Revue des patrimoines

Le cheval et ses patrimoines (1ère partie)

\title{
«Le cheval et ses patrimoines » : présentation du colloque de Tulle
}

Nicole de Blomac

\section{OpenEdition}

Journals

Édition électronique

URL : http://journals.openedition.org/insitu/9638

DOI : 10.4000/insitu.9638

ISSN : 1630-7305

Éditeur

Ministère de la culture

Référence électronique

Nicole de Blomac, « « Le cheval et ses patrimoines » : présentation du colloque de Tulle », In Situ [En ligne], 18 | 2012, mis en ligne le 31 juillet 2012, consulté le 19 avril 2019. URL : http:// journals.openedition.org/insitu/9638; DOI : 10.4000/insitu.9638

Ce document a été généré automatiquement le 19 avril 2019

\section{(c) $($ i) $(9)$}

In Situ Revues des patrimoines est mis à disposition selon les termes de la licence Creative Commons Attribution - Pas d'Utilisation Commerciale - Pas de Modification 4.0 International. 


\title{
"Le cheval et ses patrimoines»: présentation du colloque de Tulle
}

\author{
Nicole de Blomac
}

1 Nous sommes réunis ici sur un thème très particulier «Le cheval et ses patrimoines ». Pendant trois jours chacun de nous va tenter d'apporter sa contribution à une meilleure connaissance de la complexité et de la diversité de ceux-ci, ce dont je vous suis très reconnaissante comme à tous ceux grâce auxquels ce premier colloque a pu être organisé à proximité du Haras de Pompadour, notre « exception française ».

2 Il est vrai que Pompadour a joué depuis quelques années un rôle non négligeable dans la sensibilisation à ces patrimoines comme cela va vous être raconté et en découvrant la diversité de ses sites, vous comprendrez pourquoi. Ainsi, je suis sûre aussi que la visite de sa Jumenterie et de ses dépendances, où vous verrez étalons, juments et poulains arabes et anglo-arabes, vous permettra de mieux comprendre que chaque élève est un patrimoine-vivant nécessitant des architectures innovantes.

3 Pascal Liévaux ayant évoqué les grandes lignes des sujets qui vont être traités, je n'y reviens pas mais je tiens à dire combien je me réjouis de voir que c'est mon ami JeanPierre Digard, l'ethnologue, qui en tirera les conclusions et aussi que le DRAC (directeur régional des affaires culturelles du Limousin), M. Philippe Geffré, nous fera l'honneur de clôturer ce premier colloque et je ne doute pas qu'il saura en souligner tout l'intérêt culturel et patrimonial pas seulement régional.

4 Maintenant, puisque Pascal Liévaux y tient et bien que mon statut social soit bien différent des vôtres, puisque je suis seulement une retraitée de l'Agriculture et depuis peu Docteur en Histoire, je vais vous raconter comment et pourquoi je me suis tant investie, depuis si longtemps, dans le cheval et ses patrimoines. Je le fais avec plaisir tout en regrettant l'absence ici du professeur Daniel Roche, mon cher maître à qui je dois tant.

5 C'est une longue histoire dont la première étape fut mon mariage il y a plus de 55 ans avec un important agriculteur du Limousin, cette «terre de cheval» de fort ancienne réputation avec ses chevaux de selle et de sport, majoritairement de race anglo-arabe. Déjà familière du cheval, j'ai vite appris à connaître cette précieuse race de sang qui, 
grâce au Haras de Pompadour et aux écoles de dressage locales, avaient fort bien résisté à cette «fin du cheval» annoncée par celle de la Cavalerie et c'est pourquoi, initiée par quelques précieux amis, je suis moi aussi devenue éleveur d'Anglos.

Élever et surtout faire naître c'est, par le jeu des accouplements, s'inscrire dans la longue chaîne du vivant, aussi j'ai voulu en savoir plus sur les origines de mes juments et des étalons de Pompadour qui les couvraient afin de comprendre quel patrimoine vivant chacun d'eux représentait. C'est alors, qu'avec l'amicale connivence de Pierre Pechdo, alors directeur des Domaines de Pompadour puis du Haras, un collègue de mon mari pour les bovins, je me suis plongée dans les notions de filiation et de sélection sportive et que, grâce à lui, j'ai eu progressivement un accès privilégié à l'immense patrimoine écrit des Haras, à Pompadour comme à Paris et ailleurs.

7 En matière d'élevage, c'étaient il est vrai des années très dynamiques où il était aussi facile pour chacun de dialoguer avec les scientifiques, ceux qui allaient créer l'INRA et le département de génétique animale comme Bertrand Langlois, que de faire parler tous ces grands cavaliers, propriétaires et marchands, membres des syndicats d'élevage rencontrés lors des concours de chevaux.

8 Mon travail de généalogiste, publié par l'UNIC ${ }^{1}$ m'ayant fait voir l'importance de l'arabe comme composante de la race anglo, j'ai focalisé mes recherches sur son histoire en France et alors exploré de nombreuses bibliothèques et aussi les Archives nationales et cela bien avant la création du CARAN! Tout cela a débouché, avec la collaboration de Denis Bogros alors écuyer du roi du Maroc, sur L'Arabe, premier cheval de sang qui fut édité par Crépin-Leblond.

9 En fait, ce livre sur l'histoire de l'Arabe s'est avéré un bon choix car ce cheval était en train de devenir un sujet d'intérêt mondial en raison de la création en 1972 de la World Arabian Horse Organisation (la WAHO). En effet, dans ce nouveau contexte, mon travail, ma bonne connaissance de la langue anglaise et surtout la confiance que m'a alors témoignée le nouveau directeur des Haras, Henry Blanc, m'ont permis d'apporter ma contribution à ce nouvel organisme et surtout, aux côtés de Pierre Pechdo devenu le Monsieur Arabe français, de pouvoir obtenir la reconnaissance mondiale de nos Studbooks.

C'est ainsi qu'au début des années 1980, je peux dire que j'étais à la fois un éleveur engagé et assez bien coté, un membre recherché des nombreuses commissions de travail sur le cheval de sang et de sport ainsi qu'un vaillant chercheur.

11 Malheureusement, à la suite d'un grave accident personnel, j'ai dû renoncer à l'élevage et vendre tous mes anglo-arabes, ce qui fut pour moi un moment difficile. Mais heureusement, avec ce que nous avons appelé «mon testament d'éleveur », j'ai apporté ma contribution à la réalisation par le CEREOPA ${ }^{2}$, dirigé par Emmanuel Rossier, d'un ouvrage essentiel, devenu depuis un best-seller : Le cheval, techniques d'élevage.

Condamnée à abandonner l'élevage mais pas la recherche, plusieurs amis m'ont incitée alors à m'inscrire dans un cursus universitaire en histoire d'autant que ces années étaient aussi celles où le cheval était en train enfin de devenir un sujet porteur pour toute une nouvelle génération de brillants historiens, anthropologues, ethnologues, juristes, archéologues et architectes dont les travaux font référence aujourd'hui.

Grâce à Emmanuel Le Roy Ladurie, j'ai pu intégrer l'EHESS puis soutenir sous sa direction mon mémoire de maîtrise: Du coureur au galopeur, une histoire du Turf. C'était un vaste sujet que j'ai eu ensuite la chance de pouvoir développer dans La Gloire et le jeu, publié 
chez Fayard, parce qu'entre temps j'avais eu un accès privilégié à d'autres archives privées, dont celles de l'Institut de France pour la famille d'Orléans, ainsi qu'aux imposantes collections du Jockey Club.

Daniel Roche m'ayant proposé de poursuivre mon cursus avec lui, Le Roy Ladurie venant de prendre la direction de la Bibliothèque nationale de France, j'ai alors suivi soit rue d'Ulm soit au Collège de France tous ses séminaires si enrichissants en matière d'approches et d'ouvertures. Comme je venais aussi de trouver dans le personnage de Voyer d'Argenson un beau sujet de thèse qui lui plaisait, j'ai alors passé près de dix ans à travailler entre le Limousin, Paris mais aussi Poitiers où le fonds exceptionnel des archives de cette famille était alors en cours de dépôt et de classement. C'est en 2002 que j'ai soutenu ma thèse devant un brillant jury qui comportait deux membres du Collège de France puis que celle-ci fut publiée chez Belin sous le titre, Voyer d'Argenson et le cheval des Lumières.

15 Entre temps, j'avais bien entendu été sollicitée pour participer, sous des angles souvent forts différents, à de nombreux colloques, ces moments de riches échanges dont le tout premier consacré au cheval eut lieu à Avignon en 1988 à l'initiative de Jean-Pierre Digard. Comme j'en étais membre, j'ai ensuite participé à ceux si réussis de notre défunte Académie de Versailles que dirigeait Daniel Roche et dont Daniel Reytier assurait ensuite les prestigieuses publications. Depuis, il y a eu plusieurs autres colloques dont ceux organisés par Patrice Franchet d'Espèrey.

16 Mais, si les recherches que nécessitaient ces rendez-vous ne faisaient qu'exciter ma passion pour le document d'archive, elles augmentaient souvent mon désespoir devant la pauvreté des mots-clefs de certains fichiers-matières concernant le cheval et ceci aussi bien à la BnF, aux Invalides, à Vincennes ou aux Archives nationales mêmes. C'est cela qui m'avait amenée, Pascal Even ne l'a certes pas oublié, à oser m'engager dans l'aventure passionnante qui aboutira à la publication du Guide des Sources de l'histoire du cheval dans les archives publiques qu'il va vous raconter.

17 Par ailleurs, comme l'administration des Haras connaissait depuis 1999 de nombreux remaniements, j'ai alors saisi toutes les occasions de sensibiliser ses responsables à l'égard de leurs trésors patrimoniaux ce qui n'était pas évident alors. Ma première action fut d'être appelée au secours par Bernard Maurel, directeur de Pompadour, parce que le déménagement des bureaux vers le nouveau bâtiment du siège social laissait en déshérence toute la partie du château où étaient justement rassemblées les archives et la magnifique bibliothèque que je connaissais si bien. Comme sur plusieurs sites les risques de pillages étaient devenus certains, il y a eu alors une progressive prise de conscience qui a débouché sur quelques opérations essentielles au Pin et bien sûr à Pompadour comme cela va vous être raconté.

18 Certes, il a fallu du temps pour sensibiliser certains sur ces problèmes de conservation des archives du cheval mais cela a fini par déboucher en 2005 sur des réunions parisiennes très positives autour du sujet où, enfin, je n'étais plus la seule à parler en faveur de ces patrimoines.

Dans cette même perspective de conservation, une dernière mission me fut confiée par M. Roche-Bruyn, alors directeur des Haras, celle de faire un inventaire aussi exhaustif que possible de tous les livres présents dans les différents dépôts. Avec la précieuse collaboration de chacun plus celle de Sophie Lemaire pour le Pin et celle de Lucie Launay 
à Paris, j'ai pu lui en donner un compte-rendu valable dont le devenir est maintenant entre les mains de l'École nationale d'équitation à Saumur.

$\mathrm{Si}$, par ces interventions, je ne faisais que payer ma dette à la longue liste de tous ceux qui m'avaient permis d'avoir accès à tous ces ouvrages ou documents et à les utiliser pour mes travaux, il me restait pourtant une autre dette à payer, celle que j'avais à l'égard du Limousin. À l'instigation de feu notre amie Bernadette Barrière et de huit collègues historiens et archivistes régionaux, nous avons alors lancé la passionnante écriture collective de Cheval Limousin, chevaux en Limousin ouvrage édité aux Presses universitaires de Limoges.

21 Ce fut une formidable aventure qui sera tout naturellement suivie de bien d'autres et aussi de ma contribution active et toujours collective à l'tinéraire du Patrimoine consacré au Haras de Pompadour, ce petit ouvrage que vous avez en mains.

Telles furent donc les étapes d'un itinéraire fort peu banal. J'ai eu beaucoup de chance parce que ma passion du cheval-vivant et de son histoire m'a ouvert bien des portes et que j'ai pu m'y consacrer en toute indépendance et avec quelques réussites.

Comme vous avez compris, j'en suis sûre, à quel point j'ai partagé avec vous tous la passion de la recherche, de l'archive, du livre et de toutes les traces de ces patrimoines qui font l'histoire du cheval, sachez combien je me réjouis aujourd'hui de vous voir ici si nombreux pour continuer cette belle aventure, ce dont je vous remercie vivement.

\section{NOTES}

1. - The French Horse Connection.

2. - Centre d'étude et de recherche sur l'économie et l'organisation des productions animales.

\section{AUTEUR}

\section{NICOLE DE BLOMAC}

Chercheur à l'École des hautes études en sciences sociales tonyblomac@orange.fr 